Article

\title{
Dimensional Quantization and the Resonance Concept of the Low-Threshold Field Emission ${ }^{\dagger}$
}

\author{
Georgy Fursey $^{1, *}$, Pavel Konorov ${ }^{2}$, Boris Pavlov $^{3}$ and Adil Yafyasov ${ }^{2}$ \\ ${ }^{1}$ Department of Physics, University of Telecommunications, St. Petersburg 198504, Russia \\ 2 Department of Solid State Electronics, St. Petersburg State University, St. Petersburg 193232, Russia; \\ E-Mails: p.konorov@ spbu.ru (P.K.); yafyasov@gmail.com (A.Y.) \\ ${ }^{3}$ Center for Theoretical Chemistry and Physics, New Zealand Institute of Advanced Study, \\ Massey University, Auckland 0632, New Zealand; E-Mail: pavlovenator@gmail.com \\ $\dagger$ This paper is an extended version of the presentation at the International Conference on Quantum \\ Electronics, held in the St. Petersburg University of Fine Mechanics and Optics, September 2015. \\ * Author to whom correspondence should be addressed; E-Mail: g.fursey @ gmail.com; \\ Tel.: +7-921-999-02-97.
}

Academic Editor: Frank Schwierz

Received: 20 October 2015 / Accepted: 7 December 2015 / Published: 12 December 2015

\begin{abstract}
We present a brief critical review of modern theoretical interpretations of the low-threshold field emission phenomenon for metallic electrodes covered with carbon structures, taking the latest experiments into consideration, and confirming the continuity of spectrum of resonance states localized on the interface of the metallic body of the cathode and the carbon cover. Our proposal allowed us to interpret the double maxima of the emitted electron's distribution on full energy. The theoretical interpretation is presented in a previous paper which describes the $(1+1)$ model of a periodic $1 \mathrm{D}$ continuous interface. The overlapping of the double maxima may be interpreted taking into account a $2 \mathrm{D}$ superlattice periodic structure of the metal-vacuum interface, while the energy of emitted electrons lies on the overlapping spectral gaps of the interface $2 \mathrm{D}$ periodic lattice.
\end{abstract}

Keywords: low-threshold field emission; resonance states; graphene; size-quantization 


\section{Brief Review of Modern Experiments in the Low-Threshold Field Emission}

In numerous recent experiments, see for instance [1-8], extremely low-threshold field emission from the carbon nano-clusters was observed for electric fields $\left(10^{4}-10^{5} \mathrm{~V} / \mathrm{cm}\right)$. This is a surprisingly strong effect, because the field initiating a noticeable emission from these materials is 2-3 orders less than the field required for the field-emission from the traditional metals and semiconductors. Despite the obviously unusual nature of the effect, numerous authors, see for instance [6,8], have attempted to explain the low-threshold phenomenon trivially with use of the classical Fowler-Nordheim machinery, based on the enhancing of the field at the micro-protrusions. They assume that the local field $F_{s}$ near the emitting center is calculated as $F_{0}=\gamma F_{0}$, where $\gamma$ is the field enhancement coefficient, defined by the micro-geometry, and $F_{0}$ is the field of the equivalent flat capacitor. This completely classical explanation of the low -threshold emission phenomenon is not universal, and certainly non valid for carbon-covered cathodes. This has been considered in our recent papers [2,3,7] because the surface of the carbon flakes, obtained by the detonation synthesis are perfectly smooth with rare and relatively small protrusions. These protrusions are able to lower the threshold by a factor of 5, while a lowering factor of $10^{2}$ is observed in our experiments. We suggested in [2,3,7] an alternative explanation of the threshold lowering (field enhancement) based on the dimensional quantization in the under-surface space-charge region on the metal-graphene interface.

Recent experiments done by our group confirm the continuity of spectrum 2D-size quantization and allow us to estimate the effective mass $m^{*}$ and the de Broglie wavelength in space-charge region depending on electron's concentration $n_{e x}=\frac{1}{q} \int_{0}^{\tau} J(t) d t$, where $j=I / S, I=80 \mathrm{~A}$ is the current and $S \approx 0.75 \mathrm{~cm}^{2}$ - the area of the cathode, $\tau \approx 2 \times 10^{-9} \mathrm{~s}$. Monitoring of current density allows us to estimate the experimental density of charges $Q=2.4 \times 10^{-7} \mathrm{Coul} / \mathrm{cm}^{2}$, which corresponds to density of electrons $n \approx 1.3 \times 10^{12} \mathrm{~cm}^{-2}$.

On another hand, from the size-quantization theory [9] the 2D density of electrons is estimated as

$$
n_{2 D}=\frac{m^{*} k T}{2 \pi \hbar^{2}} \ln \left(1+\exp \frac{E_{0}-E_{F}}{k T}\right)
$$

Here $m^{*}$ is the effective electron mass, $E_{0}$ - the size-quantization level, $E_{F}$ - the Fermi level. In our case

$$
E_{0} \approx E_{F}, n_{\text {exp }}=n_{2 D}=\frac{m^{*} k T}{2 \pi \hbar^{2}} \ln 2
$$

The de Broglie electron wavelength [9] in graphene flakes is

$$
\lambda=\frac{2 \pi \hbar}{\sqrt{2 m^{*}(k T)}}
$$

Based on the preceding Equations (2) and (3), we estimate the electron effective mass and the de Broglie electron

$$
m^{*}=\frac{(2 \pi \hbar)^{2}}{2(k T)}
$$

At room temperature we have $\lambda \approx 18 \mathrm{~nm}, m^{*} \leq 10^{-2} m_{0}$. We should notice that the experiment based estimates yield the values $m^{*} \leq 10^{-2} m_{0}$. The above value of the electron effective mass yield the values one order higher than ones for graphene, obtained from independent experiments, see [10]. 
Another important result obtained by our group is discovery of the second maximum on the dependence of distribution of electron on total energy. This result does not have yet an adequate interpretation.

The classical Fowler-Nordheim machinery used to calculate the transmission coefficient for simple rectangular potential barrier, see [11], gives an exponentially small value of the transmission coefficient $T \approx e^{-q a}$ with $q=\sqrt{v-2 m E \hbar^{-2}}$ for the under - barrier tunneling with $v>>2 m E \hbar^{-2}$, and the width of the barrier is equal to $a$.

In our papers [2,7] we assume that the spectrum of the size quantization is discrete and reduced to several discrete levels. Based on this assumption, we developed a resonance version of the classical Fowler-Nordheim machinery, considering the complex levels as resonances. The role of field enhancing factor in our interpretation was played by the small effective mass of electron in the carbon structure. Indeed, the field is measured by the steepness of the slope of the potential. However, the effective steepness is calculated with respect to the de Broglie wavelength which is $m / m_{e}$ times bigger than the conventional de Broglie wavelength at the same energy. The corresponding formula for the transmission coefficient was derived [7] for the general 1D model of the space-charge region, with complex discrete spectrum of the surface levels.

\section{The Resonance Interpretation}

Presence of these resonance details in the barrier may result in much larger values of the transmission coefficient $T$ for electrons with certain energy. In [2] we emulated $T$ by delta-barrier supplied with inner structure defined by a differential operator on a finite interval. We realize that this differential operator does not have any physical meaning itself, but serves just as a mathematical detail of the model. Besides, fitting of the corresponding model, with this differential operator, presents some technical difficulties, in particular solving the relevant inverse problem. In [7] we base our conclusions upon a simpler model, substituting inner structure by a finite matrix, which is fit based on experimental data on size-quantization. Similarly to [2] we emulate the barrier in [7] by the generalized Datta and Das Sarma boundary condition, see [7,12,13]. The 1D solvable model of the contact zone of the emitter is constructed hereafter based on division of the normal coordinate into three layers; the metal (1) $\infty<x<0$, the vacuum (2), $a<x<\infty$ and carbon deposit (3), $0 \leq x \leq a$., with the portions of the wave-function denoted correspondingly.

The components $x_{s}, s=1,2,3$ of the wave-function of the electron satisfy on the domains the Schrödinger equations, for instance

$$
-\frac{\hbar^{2}}{2 m_{s}} \frac{d^{2} u^{s}}{d x_{s}^{2}}+q_{s}\left(x_{s}\right) u^{s}=E u^{s}, s=1,2
$$

Here $q_{1}=q_{2}=0$, end $q_{3}$ is a rectangular barrier hight $q$.

$$
u(x)=\left\{\begin{array}{ccc}
u_{1}(x)= & e^{-i p x}+T e^{i p x}, & -\infty<x<0 \\
u_{3}(x)= & B e^{\sqrt{\frac{2 m(q-E)}{\hbar^{2}}} x}+B e^{-\sqrt{\frac{2 m(q-E)}{\hbar^{2}}} x}, 0<x<a, \\
u_{2}(x)= & T e^{-i p x}, & a<x<\infty
\end{array}\right.
$$


Following [2,7], we model the component $u^{3}$ of the wave-function on the barrier-the solution of a differential equation on a finite interval, by the finite vector and, correspondingly, substitute the barrier by a zero-range model with an inner structure, see [14], defined by the finite Hermitian matrix $A$. The corresponding boundary form is represented in terms of the corresponding "boundary values" $\xi_{ \pm}^{u}$ as

$$
J^{s}(u, v)=\frac{\hbar^{2}}{2 m_{s}}\left[\frac{d u^{s}}{d x^{s}} \bar{v}^{s}-u^{s} \frac{d \bar{v}}{d x^{s}}\right], s=1,2
$$

The corresponding boundary forms of the inner structure in terms of the adequate boundary values is

$$
J^{3}(u, v)=\xi_{+}^{u} \bar{\xi}_{-}^{v}-\xi_{-}^{u} \bar{\xi}_{+}^{v}
$$

The zero-range model of the resonance field emission is defined by the boundary condition imposed on the data, which annihilate the sum of the boundary forms $J^{1}(u, v)+J^{2}\left(u^{\prime} v\right)+J^{3}(u, v)$. When discussing the zero-range model of the interface lattice, we assume, that the deposit zone is substituted by the model barrier, but the zone inherits the small effective mass $m_{1} \leq m_{2}$ from the deposit on the contact interface. In particular the sum is vanishing while the boundary data are imposed to the generalized Datta-Das Sarma boundary condition at the contact of the deposit and vacuum (that is exactly on the barrier). This boundary condition is defined, similarly to Datta-Das Sarma, [12], by the vector parameter $\vec{\beta}=\left(\beta_{1}, \beta_{2}, \beta_{3}\right)$ as:

$$
\frac{u^{1}}{\beta_{1}}=\frac{u^{2}}{\beta_{2}}=\frac{\xi_{-}^{u}}{\beta_{1}}, \frac{\hbar^{2}}{2 m_{1}} u_{1}^{\prime} \bar{\beta}_{1}+\frac{\hbar^{2}}{2 m_{2}} u_{2}^{\prime} \bar{\beta}_{2}+\xi_{+}^{u} \bar{\beta}=0
$$

The quantum-mechanical meaning of the similar parameter $\vec{\beta}$ in the case of T-junction is revealed in [12]. In our case, the parameter is defined by the geometry of the contact zone. We assume here that it can be fitted based on the experimental data. The boundary values $\xi_{ \pm}^{u}$ of the component of the wave-function on the barrier are connected via the corresponding Weyl-Titchmarsh function $\mathcal{M}(E)=P_{N} \frac{I+E A}{A-E I} P_{N}$, and selected "deficiency" subspace $N$, defining the connection of the inner space (the space of the size-quantization on the barrier), with the vacuum and the deposit. The energy $E$ plays a role of the spectral parameter, so that the dimension of the operator $A$ is energy. Hereafter, we assume that the Weyl function $\mathcal{M}(E)$ is scalar, but our model can be easily extended to the general case with multidimensional Weyl function. Assuming that the wave-function of the electron in the deposit and in vacuum is a scattered wave, we represent the components of it in the deposit and in vacuum as $u_{1}=e^{i p x}+e^{-i p x} R_{1}, u_{2}=T_{2} e^{-i p x}$. Substituting this scattering Ansatz into the above boundary conditions, we obtain an expression for the transmission coefficient $T$ from the deposit into vacuum :

$$
T(\lambda)=\frac{\bar{\beta}_{1} \beta_{2} m_{1}^{-1 / 2}}{\left|\beta_{1}^{2}\right| m_{1}{ }^{-1 / 2}+\left|\beta_{2}^{2}\right| m_{2}^{-1 / 2}+i\left|\beta_{3}\right|^{2}[\hbar \mathcal{M} \sqrt{2 E}]^{-1}}
$$

In the non-resonance situation, $\mathcal{M} \approx$ Const, the Datta-Das Sarma parameter $\left(1,1, e^{q a / 2}\right)$ defines the exponential small transmission rate $T \approx e^{-a q}$. Then, in the resonance situation, $\mathcal{M}=\mathcal{M}(\lambda)$ the transmission is exponentially small on the complement of the set of poles of $\mathcal{M}$, but is essentially greater at the poles $\lambda_{p}, \mathcal{M}\left(\lambda_{p}\right)=\infty$, where $\mathcal{M}^{-1}\left(\lambda_{p}\right)=0$. With regard of $m_{1}<<m_{2}$ we have at the poles, that

$$
T=\frac{\bar{\beta}_{1} \beta_{2} m_{1}^{-1 / 2}}{\left|\beta_{1}\right|^{2} m_{1}^{-1 / 2}+\left|\beta_{2}\right|^{2} m_{2}^{-1 / 2}} \approx \frac{\beta_{2}}{\beta_{1}}
$$


which can be essentially greater than exponential estimate $T \approx e^{-2 a v}$. Then, in the resonance situation, $\mathcal{M}=\mathcal{M}(\lambda)$ based on $\beta_{3} \approx e^{v d}$ we see the peak of the transmission coefficient at the eigenvalues of the matrix $A$ which play a role of the levels of the size - quantization on the barrier (with special boundary conditions on the contact of the barrier with the inner part of the deposit and the vacuum. This condition is compatible with unitarity of the full scattering matrix on the interface deposit-vacuum, if the weights $m_{1}^{-1}, m_{2}^{-1}$ are taken into account.

In the ballistic regime, the current $j$ is proportional to the weighted integral of the transmission coefficient $T$. In particular, for linear spectrum $E=\hbar v_{F} k$ the integration is performed on the energy variable

$$
j=-\frac{2 e v_{F}}{\hbar^{2}} \int T(E)[f(E)-f(E+e V)] E d E
$$

Here, $\mathrm{eV}$ is the voltage drop, $f(E)$ is the Fermi distribution, and $v_{F}$ is the Fermi velocity. For low temperature the integrand with the Fermi distribution is reduced to

$$
[f(E)-f(E+e V)] \approx-e V \delta\left(E-E_{F}\right)
$$

In our case we have generally:

$$
j=-\frac{2 e v_{F}}{\hbar^{2}} \int \frac{\bar{\beta}_{1} \beta_{2} m_{1}^{-1 / 2}[f(E)-f(E+e V)] E d E}{\left|\beta_{1}\right|^{2} m_{1}^{-1 / 2}+\left|\beta_{2}\right|^{2} m_{2}^{-1 / 2}+i\left|\beta_{2}\right|^{2}[\hbar \sqrt{2 E} \mathcal{M}]^{-1}}
$$

with $\mathcal{M}=\mathcal{M}(E)$, and, for low temperature, just the value of $T$ at the Fermi level, with a trivial coefficient.

\section{Discussion: The Role of the Interface Structure}

The above model of resonance tunneling through the size - quantization level presents a qualitative interpretation the low-threshold emission. The magnitude of the threshold measured in experiment (see [2,3]) is $q V=(20-100) \mathrm{meV}$. These values of the threshold may be qualitatively explained based on resonance tunneling through the size-quantization level. Up to choice of the statistical distribution, this magnitude corresponds to $q V=E_{0}-E_{F}=(2-3) k T$, for room temperature.

The reason for this may have various causes. First of all: the one-dimensional version of the electron transmission through the interface barrier (even with regard to size-quantization levels). Another cause is absence of theoretical equivalent of the multilayer structure in the accepted model of the cathode, usually substituted by the two-layer structure of the contact of graphene and the metal surface, without taking into account mutual influence of the components. Finally, the naive model does not take into account the two-dimensional structure of the graphene-metal interface, which may play an essential role for both classical Fowler-Nordheim or resonance transmission scenario of the field emission. In any case, the enhanced values of the measured electron mass may be interpreted as evidence of the emission from narrow spectral bands, rather than one from the discrete resonance levels. Even considering the simplest $(1+1)$ model of the graphene-metal interface, we discover that the electron mass may be greater than in pure graphene due to possible level crossings arising from the dispersion curves of the materials involved - the graphene and the metal. Even a naive $(1+1)$ model of the graphene-metal interface 
shows the levels crossings and relevant super-narrow spectral bands and gaps with various values of effective electron mass.

Indeed, let us observe the influence of periodicity of the graphene-metal interface in the simplest case $(1+1)$ of $1 \mathrm{D}$ of the periodic structure of the interface. Using a discrete model of a 1D periodic graphene-metal superlattice, we obtain the dispersion function of the lattice, with relevant Weyl-Titchmarsh function $M(\lambda)$, taking into account an interaction only between the nearest neighbors in the lattice:

$$
\mathcal{M}(\lambda)-B \cos p=0
$$

Here, the Weyl-Titchmarsh function of the graphene-metal superlattice is composed of the functions of component lattices and the interaction is taken into account by $2 \times 2$ matrix $B$. The effective mass of electron in the resulting interface lattice does not coincide with the mass in each of component lattices, but depends on the interaction $\beta$. Generally, it may be greater than the mass of electron in graphene. The Weyl-Titchmarsh function has a positive imaginary part in the upper half-plane of energy $\Im \lambda \geq 0$ and real on the spectral gaps of the lattice. Then the effective mass of the electron on the lattice is calculated as $\left[\frac{d \lambda^{2}}{d p^{2}}\right]$, which yields, at the ends of the spectral bands, where $\left[\frac{d \lambda}{d p}\right]=0$

$$
m=-\frac{\frac{d \mathcal{M}}{d \lambda}}{\beta \cos p}=-\frac{\frac{d \mathcal{M}}{d \lambda}}{\mathcal{M}(\lambda)}
$$

Based on effective mass we may judge on density of states which allows to estimate the emission current based on above formulae for the transmission coefficient Equation (8). While calculating the transmission coefficient with regard of 1D periodic structure of the graphene-metal interface,

$$
T(\lambda)=\frac{2 \pi i}{2 \pi i+\mathcal{M}(\lambda)}
$$

we notice that the transmission coefficient may be large on the gaps of the interface-lattice and relatively small on the open spectral bands of the interface. For the 2D interface, two spectral bands may overlap, yielding the resulting picture with two maxima in the electron's distribution on full energy, see [1]. We plan to continue our study on the corresponding phenomena in our upcoming publications, based on 2D mathematical tools prepared in our preceding papers, see for instance [13].

\section{Conclusions}

The paper provides a brief overview of the main results of an experimental study of field emission from the graphene-like structures, which implies that the emissions are of a low-threshold character.

One-dimensional theoretical model of the issue, based on the resonant tunneling effect, is analyzed. The advantages and disadvantages of the one-dimensional model are discussed.

\section{Acknowledgments}

We are grateful to V. Bogevolnov, who attracted our attention to this cheap and highly interesting material and supplied us with a sufficient amount of it and to M. Polyakov for experimental dates of pulse studies. 


\section{Author Contributions}

The article is written by authors in the creative equivalent to participation in the creation of the text in the presentation and discussion of the results.

\section{Conflicts of Interest}

The authors declare no conflict of interest.

\section{References}

1. Fursey, G.N.; Egorov, N.V.; Zakirov, I.I.; Yafyasov A.M.; Antonova L.I,; Trofimov V.V. Peculiarities of the total energy distribution of the field emission electrons from graphene-like structures. Radiotech. Electron. 2016, 61, 79-82, in press. (In Russian)

2. Yafyasov,A; Bogevolnov, V.; Fursey, G.; Pavlov, B.; Polyakov, M.; Ibragimov, A. Low-threshold emission from carbon nano-structures In: Ultramicroscopy 2011, 111, 409-414.

3. Fursey, G.N.; Polyakov, M. A.; Kantonistov, A.A.,; Yafyasov, A. M.; Pavlov, B. S.; Bogevolnov, V. B. Field and Explosive Emission from Graphene-Like Structures. Tech. Phys. 2013, 58, 845-851.

4. Fursey, G.N. Field Emission in Vacuum Microelectronics; Springer, Kluwer Academic/Plenum: New York, NY, USA, 2005; pp.161-168.

5. Fursey, G.N. Field emission in vacuum micro-electronics. Appl. Surf. Sci. 2003, 215, 1113-1134.

6. Forbes,G.; Xanthakis, J.P. Field penetration into amorphous-carbon films: Consequences for field-induced electron emission. Surf. Interface Anal. 2007, 39, 139-145.

7. Fursey, G.N.; Polyakov, M.; Pavlov, B.; Yafyasov, A.; Bogevolnov, V. Exceptionally low Threshold of Field Emission from carbon nano-clusters. Extended abstract. In Proceedings of the 24th International Vacuum Nanoelectronics Conference, Bergishe Universitat, Wuppertal, Germany, 18-22 July 2011.

8. Modinos, A.; Xanthakis, J.P. Electron emission from amorphous carbon nitride film. Appl. Phys. Lett. 1998, 73, 28-37.

9. Davies, J.H. The Physics of Low-Dimensional Semiconductor; An Introduction; Cambridge University Press: Cambridge, UK, 1998; p. 438.

10. Novoselov, K.S.; Geim, A.K.; Morozov, S.V.; Jiang, D.; Katsnelson, M.I.; Grigorieva, I.V.; Dubonos, S.V.; Firsov, A.A. Two-dimensional gas of massless Dirac electrons in graphene. Nature 2005, 438, 197-200.

11. Flugge, S. Practical Quantum Mechanics I. Springer Verlag, Heidelberg: New York, NY, USA, 1974.

12. Harmer, M.; Pavlov, B.; Yafyasov, A. Boundary condition at the junction. J. Comput. Electron. 2007, 6, 153-157.

13. Bagraev, N.; Martin, G.; Pavlov, B.; Yafyasov, A.; Goncharov, L.; Zubkova, A. The Dispersion Function of a Quasi-2D Periodic Sandwich via DN-Map. In Proceedings of the 11th International Conference on Computational Structures Technology, Dubrovnik, Croatia, 4-7 September 2012; Barry, H.N., Ed.; Topping, Heriot-Watt University: Edinburgh, UK, 2012; p. 53. 
14. Pavlov, B. The theory of extensions and explicitly-soluble models. Russian Math. Surv. 1987, 42, 127-168.

(c) 2015 by the authors; licensee MDPI, Basel, Switzerland. This article is an open access article distributed under the terms and conditions of the Creative Commons Attribution license (http://creativecommons.org/licenses/by/4.0/). 